\title{
Crosstalk and image uniformity in passive matrix polymer LED displays
}

\author{
D. Braun ${ }^{a}$, J. Rowe ${ }^{a}, G . \mathrm{Yu}^{\mathrm{b}}$ \\ ${ }^{a}$ Electrical Engineering Department, Cal Poly State University, San Luis Obispo, California, 93407, U.S.A. \\ ${ }^{h}$ UNIAX Corporation, Santa Barbara, Califormia, 93117, U.S.A.
}

\begin{abstract}
Passive matrix displays based on polymer and organic light-emitting diodes suffer from crosstalk, when the emission of light from one pixel depends on the operation of another pixel. We use circuit simulation in order to explain the causes of crosstalk and to quantify the consequences. This work analyzes how crosstalk arises from electrode resistance, pixel leakage current, and the location of faulty pixels. All factors influence both image uniformity and the power consumption of the display.
\end{abstract}

Keywords: Electroluminescent displays, Poly(phenylene vinylene) derivatives, Electrical circuit simulation

\section{Introduction}

Much interest in polymer and organic light-emitting diodes [LEDs] derives from their potential to deliver the next generation of tlat passive matrix displays [1-4]. The devices are relatively easy to make, because they consist of an electroluminescent layer sandwiched between an anode, usually transparent, and a cathode. A passive matrix display results by patterning the anode into columns and the cathode into rows to form an array of pixels from the intersections between the cathode and anode electrodes. Applying a positive bias to one anode and a negative bias to one cathode causes light emission from the pixel shared by the two electrodes. In many cases, activating one pixel influences light emission from one or more other pixels. The crosstalk has several origins. Earlier work describes how crosstalk depends on display resolution, the degree of rectification of the individual LEDs, and, most significantly, on the reverse leakage current of the pixels [5]. This work explores how electrode resistance and the location of faulty pixels can degrade image uniformity in row scanned passive matrix displays.

\section{Electrode resistance}

Fig. 2 shows how resistance in the row electrode decreases imuge uniformity along one row. The approach used is to insert the electrical data for $1 \mathrm{~cm}^{2}$ LEDs from Fig. 1 directly into the PSpice circuit simulation tool along with a circuit net list for the 10 pixel row circuit shown in the inset to Fig. 2. Each pixel contains one LED and a row (cathode) resistance of $10 \mathrm{~m} \Omega /$ pixel.

Fig. 3 shows how resistance in the column (anode) electrode decreases image uniformity along one column. The circuit in the inset to Fig. 3 illustrates that the top pixel in the column sees a larger series resistance than the bottom pixel and therefore receives a smaller current density at the same applied bias.

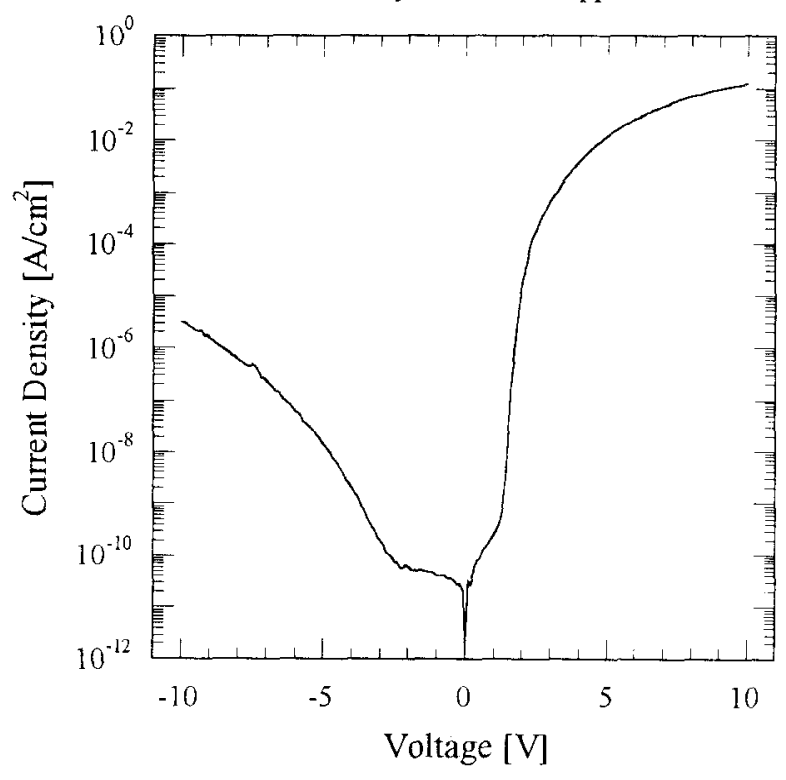

Fig. 1. Current as a function of voltage recorded for a polymer LED made from MEH-PPV.

\section{Location of pixel faults}

In Fig. 4 , the location of a leaky pixel in a row determines the severity of non-uniformity. With a pixel fault of $100 \Omega$ in the pixel closest to the voltage source, the first pixel diverts the 
excess current, so other pixels in the row suffer only a minor drop in intensity. A pixel fault further down the row sends the excess current further down the row, creating a larger voltage drop along the cathode clectrode and dropping the intensity of more pixels in the row

\section{Conclusion}

Degraded image uniformity due to electrode resistance implies that drivers should supply current instead of voltage to column contacts. In addition, electrode resistance increases the power consumption of the display. Because the problems increase with drive current, higher efficiency LEDs can also decrease problems of uniformity and power consumption that arise from electrode resistance and pixel faults. Quantitatively, the above simulation results based on rows and columns 10 pixels long illustrate that a $100 \mathrm{~cm}^{2}$ display will suffer dramatically from poor uniformity

\section{References}

[1] P. May, SW 96 Digest, (1996) 192-195.

[2] J. R. Sheats, H. Antoniadis, M. Hueschen, W. Leonard, J Miller, R. Moon, D. Roitman, and A. Stocking, Science, 273 (1996) 884-888

[3] A. Shen, P. E. Burrows, and M. E. Thompson, Science, 276 (1997) 2009

14] Proceedings of the 1996 Intemational Conference on Science and Technology of Synthetic Metals, Synth. Met., 85 (1997) 1173-1444.

[5] D. Braun, Synth. Met, 92 (1998) 107-113

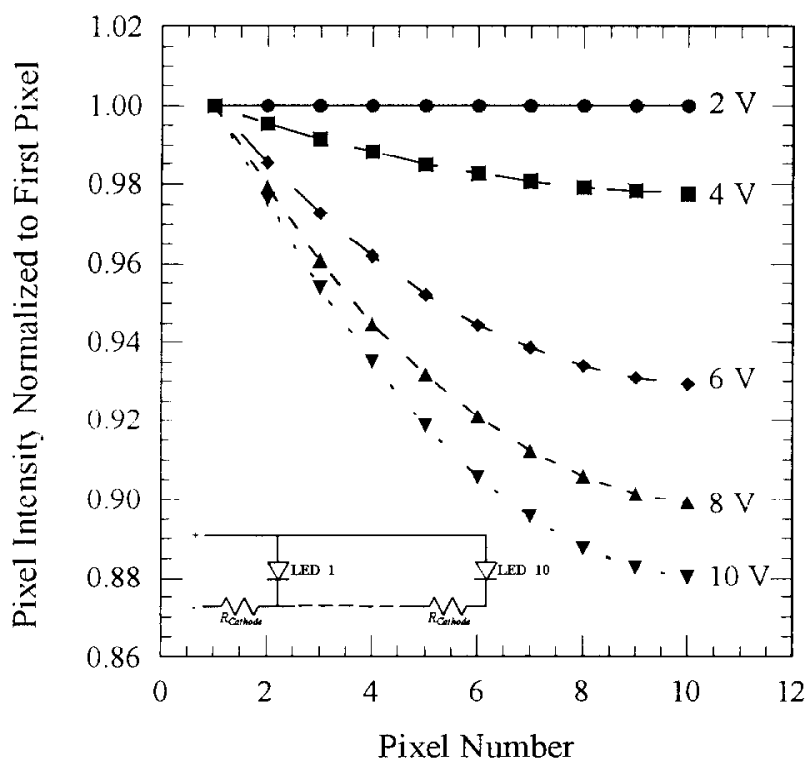

Fig. 2. Pixel intensity in one row as a function of pixel position with applied bias ranging from $2 \mathrm{~V}$ to $10 \mathrm{~V}$. The row (cathode) resistance is $10 \mathrm{~m} \Omega /$ pixel. With the anode of each pixel in the row biased to the same voltagc, current flow in the row clectrode produces voltage drops that create unequal voltages at the cathodes of each pixel.

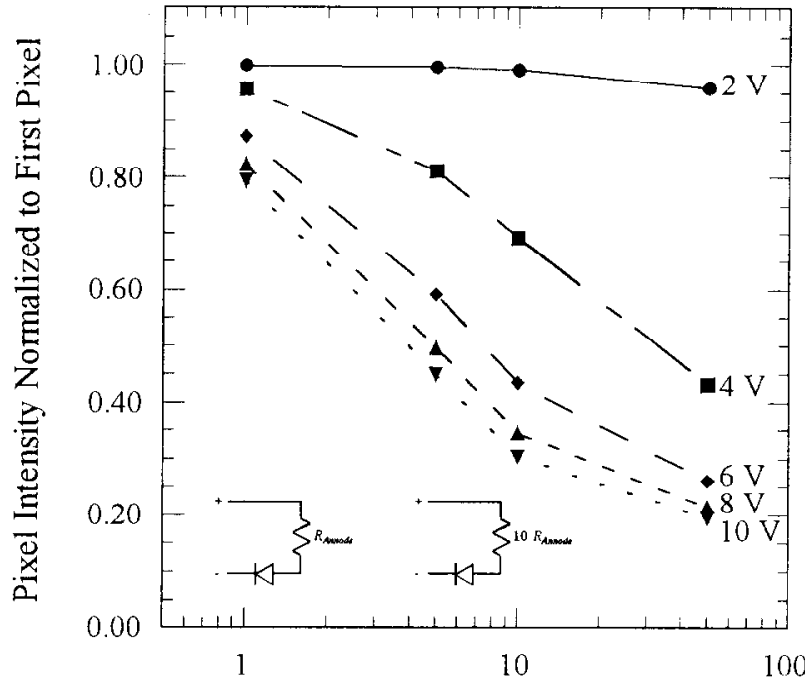

Column Resistance $[\Omega /$ pixel]

Fig. 3. Pixel intensity ratio in one column as a function of column (anode) resistance and applied bias. In a column of 10 pixels, the anode resistance of the top pixel is 10 times that of the bottom pixel.

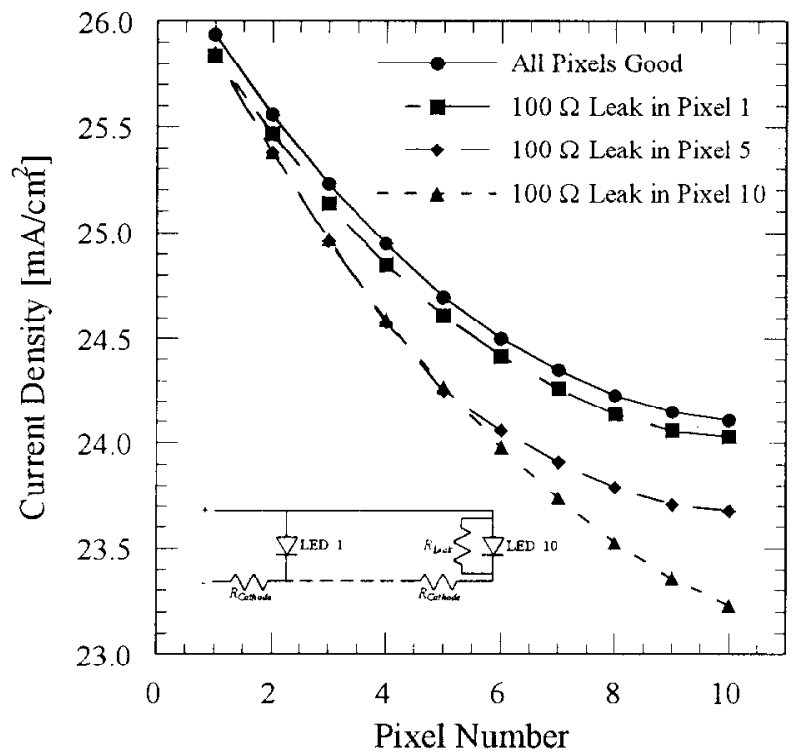

Fig. 4. Pixel intensity in one row as a function of pixel position and a faulty pixel. The row (cathode) resistance is $10 \mathrm{~m} \Omega / \mathrm{pixel}$, and each cathode has a bias of $6 \mathrm{~V}$. With the anode of each pixel in the row biased to the same voltage, current flow in the row electrode produces voltage drops that create unequal voltages at the cathodes of each pixel. The voltage drops are most severe "upstream" of the leak. 